NIST Technical Note 2149

\title{
UE-to-Network Relay Model B Discovery in ProSe-Enabled LTE Networks
}

Alexandre Moreaux

Samantha Gamboa

David Griffith

Richard Rouil

This publication is available free of charge from:

https://doi.org/10.6028/NIST.TN.2149

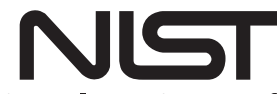

National Institute of Standards and Technology

U.S. Department of Commerce 


\title{
NIST Technical Note 2149
}

\section{UE-to-Network Relay Model B Discovery in ProSe-Enabled LTE Networks}

\author{
Alexandre Moreaux \\ Associate, Wireless Networks Division \\ Communications Technology Laboratory \\ Samantha Gamboa \\ Associate, Wireless Networks Division \\ Communications Technology Laboratory \\ Prometheus Computing LLC \\ Sylva, North Carolina \\ David Griffith \\ Richard Rouil \\ Wireless Networks Division \\ Communications Technology Laboratory
}

This publication is available free of charge from: https://doi.org/10.6028/NIST.TN.2149

April 2021

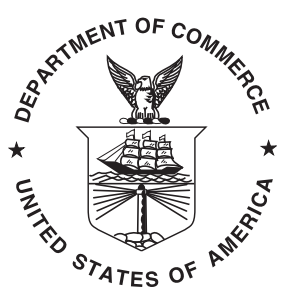

U.S. Department of Commerce Gina M. Raimondo, Secretary

National Institute of Standards and Technology James K. Olthoff, Performing the Non-Exclusive Functions and Duties of the Under Secretary of Commerce for Standards and Technology \& Director, National Institute of Standards and Technology 
Certain commercial entities, equipment, or materials may be identified in this document in order to describe an experimental procedure or concept adequately. Such identification is not intended to imply recommendation or endorsement by the National Institute of Standards and Technology, nor is it intended to imply that the entities, materials, or equipment are necessarily the best available for the purpose.

National Institute of Standards and Technology Technical Note 2149

Natl. Inst. Stand. Technol. Tech. Note 2149, 19 pages (April 2021)

CODEN: NTNOEF

This publication is available free of charge from:

https://doi.org/10.6028/NIST.TN.2149 


\begin{abstract}
In this document, we expand previous analytical models to offer a more complete representation of Model B direct discovery protocol used for Proximity Services (ProSe) User Equipment (UE) relay functionality, called UE-to-Network Relay. Using these models, we can obtain performance metrics such as the average time a given Remote UE takes to discover a Relay UE, and we can examine the sensitivity of the discovery performance to various input parameters such as the number of Remote UEs, the number of Relay UEs, and the discovery message transmission rate. We use ns-3 system-level simulations to validate our analytical model and we demonstrate the performance of the UE-to-Network Relay discovery protocol for different values of the discovery message transmission probability, which is a system parameter that the UE can use to throttle its discovery message transmission rate to reduce the probability of collisions.
\end{abstract}

\title{
Key words
}

D2D; Discovery; LTE; Markov Chain; Network Modeling; ProSe; UE-to-Network Relay. 


\section{Table of Contents}

1 Introduction 1

2 Analytical Model $\quad 3$

2.1 System Model 3

2.1.1 Scenario 3

2.1.2 Message Transmission 4

2.1.3 Metric of Interest 4

2.1.4 Additional Assumptions 5

2.2 Analytical Derivation 5

2.2.1 Markov Chain $\quad 5$

2.2.2 State Transition Probabilities 8

3 Numerical Results $\quad 11$

3.1 System-Level Simulations $\quad 11$

3.2 Result Discussion 11

4 Conclusions and Future Work $\quad 13$

$\begin{array}{ll}\text { References } & 14\end{array}$

\section{List of Tables}

Table 1 List of symbols

\section{List of Figures}

Fig. 1 The discovery resource pool model, showing the transmissions of various UEs and indicating the location of $X$ 's discovery message $\delta_{X}$ and the subframes it occupies $S_{X}$ [1, Fig. 3].

Fig. 2 Markov chain modeling the system in study.

Fig. 3 Average number of discovery periods needed by a Remote UE to discover a given Relay UE $(t(X \circ Y))$. Mean and $95 \%$ confidence intervals are shown for the system-level simulation results. 


\section{Introduction}

Release 12 of the 3rd Generation Partnership Project's (3GPP's) Long Term Evolution (LTE) standard introduced procedures for Device-to-Device (D2D) communications between User Equipment (UE) enabling Proximity-based Services (ProSe). One of the applications of ProSe is Public Safety communications in environments where a direct link to an eNodeB (eNB) (i.e., a base station) is not possible. Allowing devices to send information directly to each other without relying on eNBs will give first responders the capability to communicate in environments with limited or unavailable cellular coverage, such as deep indoor areas, uninhabited and remote areas or disaster areas where the cellular infrastructure has been degraded or destroyed. D2D communications between UEs take place over a set of time-frequency resources known as the sidelink (SL), which is distinct from the eNB-to-UE downlink (DL) and the UE-to-eNB uplink (UL). An important feature of ProSe technology is the UE-to-Network Relay functionality that 3GPP introduced in Release 13 of the LTE standard. This function allows some in-coverage UEs (Relay UEs) to use the SL to ferry traffic between the eNB and UEs that are close enough to a Relay UE to communicate via the SL but which are outside the eNB's coverage area (Remote UEs).

The first step for a Remote UE that intends to use the UE-to-Network Relay function is to use ProSe's direct discovery functionality to search for suitable nearby Relay UEs. The 3GPP specifications define two ProSe direct discovery models: Model A and Model B [2]. Model A is a one-way discovery process where Relay UEs broadcast discovery messages that inform nearby Remote UEs of their presence and their connectivity features. Remote UEs that receive discovery messages from Relay UEs can use the information in the discovery messages to select a Relay UE and to establish a relay connection over the SL. In contrast, Model B is a two-way discovery process where Remote UEs broadcast solicitation messages to Relay UEs. Relay UEs that receive solicitation messages attempt to send response messages that, if successfully received by the Remote UE, allow the Remote UE to obtain the Relay UEs' information in order to select a relay and establish a connection to it. For either discovery model, a Remote UE that has discovered available Relay UEs selects which Relay UE to use based on a variety of factors (e.g., signal strength) and starts the one-to-one ProSe direct communication procedure to connect to the selected Relay UE [3].

The LTE standard does not define which discovery model to use. Both models have advantages and it is up to network operators to choose the configuration parameters that fulfill their requirements. For example, Gamboa et al. have shown that Model A allows faster discovery than Model B for equivalent network configurations, but Relay UEs using Model B transmit fewer messages and hence consume less energy than Relay UEs that use Model A, which requires them to continuously transmit discovery messages [4]. Analytical and simulation models are valuable tools for evaluating such design trade-offs and for planning public safety network deployments.

ProSe-enabled LTE UEs use the Physical Sidelink Discovery Channel (PSDCH) to broadcast discovery messages on the SL. During each discovery period, UEs select timefrequency resources out of a discovery pool to transmit discovery messages [5]. The dis- 
covery pool is constituted of Physical Resource Block (PRB) pairs and subframe sets. The allocation of these resources can be scheduled by an eNB, if the UEs are in its coverage area, or randomly selected by the UEs themselves, referred to as UE-selected scheduling; out-of-coverage UEs always use UE-selected scheduling and preconfigured pool parameters. When using UE-selected scheduling, and because resource allocation is random in the absence of a central synchronizing entity, packet collisions will occur if multiple UEs pick the same resources, and discovery messages may be lost. To circumvent the issue, 3GPP defined a transmission probability parameter, txProbability, that throttles UE transmissions by making UEs generate a random number which they compare to txProbability to decide whether to transmit in each discovery period. Multiple researches have focused on direct discovery scheduling. Interested readers can refer to the related work section in [6] and references therein for a comprehensive selection.

In [6], we focused on the UE-selected discovery resource allocation, as eNB scheduling information may not be available for the Remote UEs in the UE-to-Network Relay architecture. We developed an analytical model to quantify the time taken by a given Remote UE to discover (i) any Relay UE in proximity, and (ii) a specific Relay UE in proximity, depending on the discovery pool configuration and the discovery model used by the UEs. We assumed that Remote UEs are synchronized when using Model B, i.e., all Remote UEs in the system transmit solicitations in the same discovery period and wait for responses in the next period, avoiding resource contention with Relay UE responses. In practice, Remote UEs can start the discovery procedure in any discovery period and thus contend for discovery resources with Remote UEs and Relays UEs indistinctly. Moreover, the half-duplex effect prevents the UEs from transmitting and listening for discovery messages at the same time, which causes that Relay UEs could miss solicitations when transmitting a response, and Remote UEs could miss responses when transmitting a solicitation.

In this document, we build upon the model in [6] to construct a more complete analytical model for direct discovery Model B. In this analysis we consider that Remote UEs can start the discovery process at different discovery periods and we take into account the resulting impact in the model. Our analysis develops an expression for the average time a given Remote UE takes to discover a given Relay UE, and our model includes the effect of the pool configuration, including the txProbability parameter, and also incorporates the impact of message collisions and the half-duplex effect in direct discovery Model B.

The analytical model developed in this document was validated using system-level simulations performed using the ns-3 ProSe module described in [7] and enhanced with the UE-to-Network Relay functionality in [4]. Therefore, it allows network planners to examine the impact of input parameters of interest and identify trends using only a fraction of the computational resources required by simulations.

The rest of the document is structured as follows. Section 2 develops the analytical model and derives an expression for the average time a given Remote UE takes to discover a given Relay UE. Section 3 includes numerical results from the analytical model and simulation model and discusses the sensitivity of the above metric to various parameters. Finally, Section 4 summarizes our contributions and discusses future work. 


\section{Analytical Model}

In this section, we develop the analytical model that gives the average time that a given Remote UE takes to discover a given Relay UE when using Model B ProSe direct discovery. We define the system in Section 2.1, and we develop the model and derive the expression for the average relay discovery time in Section 2.2. A list of the symbols that we use throughout this document is in Table 1 .

\subsection{System Model}

\subsubsection{Scenario}

We consider two mutually exclusive groups of UEs: group $G$ consists of $N_{x}$ Remote UEs and group $H$ consists of $N_{y}$ Relay UEs, as in [6]. All UEs are located within each other's respective reception ranges and are randomly deployed according to a uniform distribution. Let $X$ be a given Remote UE and let $Y$ be a given Relay UE that are randomly chosen from groups $G$ and $H$ respectively.

Table 1. List of symbols

\begin{tabular}{c|l}
\hline Symbol & Definition \\
\hline$P(A)$ & Probability of event A \\
$G$ & The set of Remote UEs in context \\
$N_{x}$ & card $(G)$ \\
$N_{x 1}$ & card $\left(G_{1}\right)$ \\
$N_{x 2}$ & card $\left(G_{2}\right)$ \\
$\mathrm{UE} X$ & Randomly chosen Remote UE of interest from $G$ \\
$H$ & The set of Relay UEs in context \\
$N_{y}$ & card $(H)$ \\
$\mathrm{UE} Y$ & Randomly chosen Relay UE of interest from $H$ \\
$\delta_{X}$ & Discovery message sent by UE $X$ \\
$S_{X}$ & Set of subframes occupied by $\delta_{X}$ \\
$N_{r}$ & Number of resources in discovery pool \\
$N_{f}$ & Number of PRB pairs in discovery pool \\
$N_{t}$ & Number subframe sets in discovery pool \\
$\theta$ & Probability that a given UE transmits \\
$T$ & Markov state transition matrix \\
$N$ & $T$ 's Fundamental Matrix \\
$Z_{1}, Z_{2}$ & Arbitrary UEs \\
$G_{1 / 2} \rightarrow Z_{2}$ & $G_{1 / 2}$ successfully sends a query to $Z_{2}$ \\
$Z_{2} \sim Z_{1}$ & $Z_{2}$ responds to $Z_{1}$ \\
$Z_{1} o Z_{2}$ & $\left(Z_{1}\right.$ discovers $\left.Z_{2}\right)=\left(Z_{1} \rightarrow Z_{2}\right) \cap\left(Z_{2} \sim Z_{1}\right)$ \\
\hline
\end{tabular}




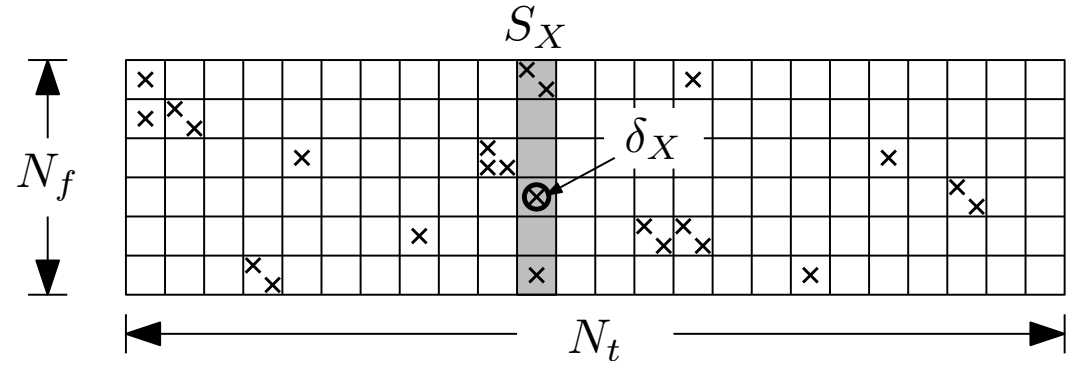

Fig. 1. The discovery resource pool model, showing the transmissions of various UEs and indicating the location of $X$ 's discovery message $\delta_{X}$ and the subframes it occupies $S_{X}$ [1, Fig. 3].

\subsubsection{Message Transmission}

During each discovery period, UEs randomly select a resource in which to transmit from the discovery resource pool, which we model as a $N_{f} \times N_{t}$ matrix, as shown in Fig. 1. Before transmitting in its selected discovery resource pool resource, each UE with a discovery message to send generates a uniformly distributed random value $p 1 \in[0,1]$ that it compares with the system parameter txProbability. The UE will not transmit its discovery message unless $p 1$ is less than or equal to txProbability. This transmission probability thus acts as a simple throttling mechanism that can reduce the offered load from UEs and help prevent discovery message collisions. Let $\theta$ be the probability that a UE transmits in a given discovery period, i.e., $\theta=P(p 1 \leqslant t x$ Probability $)$.

Unlike our previous work in [6], we consider the case that not all Remote UEs in group $G$ send solicitation messages in the same discovery period. In other words, each Remote UE randomly determines whether its starting state is "active" or "inactive". Remote UEs that are in the "active" state in a given discovery period attempt to transmit discovery messages in that period; they enter the "inactive" (i.e., sleep) state during the following discovery period in order to receive possible response messages from Relay UEs. We number the discovery periods during the time interval that we are modeling; we let $G_{1} \subseteq G$ be the subgroup of Remote UEs that are active during the first period, and we let $G_{2} \subseteq G$ be the subgroup of Remote UEs that are inactive during the first discovery period.

\subsubsection{Metric of Interest}

In the analysis in this subsection, we derive the average time for a given Remote UE to discover a given Relay UE. In ProSe direct discovery Model B, a discovery is successful when a Remote UE receives a response message from a Relay UE. The Relay UE transmits the response message in response to receiving one or more solicitation message from Remote UEs during the previous discovery period. All UE discovery message transmissions (i.e., solicitations and responses) are broadcast to all UEs in range, and successfully received response messages from Relay UEs cause discoveries for any Remote UE receiving them even if that Remote UE is not the source of the original solicitation message. Discovery message transmissions can fail due to packet collisions or due to the half-duplex effect, 
where UEs are unable to receive messages during the subframes in which they transmit.

Given that we are studying a closed system whose state changes at regularly distributed intervals, it is natural to adopt a state machine approach to modeling it. We define the principal events for a Remote UE as follows. Let

- $X \rightarrow Y$ : Remote UE $X$ successfully sends a solicitation message to Relay UE $Y$

- $Y \rightarrow X$ : Relay UE Y successfully sends a response message to Remote UE X

- $X \circ Y$ : Remote UE $X$ discovers Relay UEY

We are interested in the average time for a given Remote UE $X$ to discover a given Relay UE $Y$, which we denote as $t(X \circ Y)$.

\subsubsection{Additional Assumptions}

We assume that all UEs belong to the same security domain and are authorized to perform UE-to-Network Relay discovery. This approach could be deemed Open Access although its long-term applications are focused on Restricted Access in the Public Safety context.

We assume that when multiple UEs transmit messages using the same discovery pool resource, all the colliding transmissions are lost. In reality, one or more of these transmissions can be recovered if the Signal-to-Interference-plus-Noise Ratio (SINR) is high enough, but we make the conservative assumption that they are all lost.

We also assume a worst-case transmission scenario where the Remote UEs other than the UE of interest always send new solicitations, even after a successful discovery. This assumes that each Remote UE constantly monitors the discovery resource pool for response messages from Relay UEs that can provide better connectivity than the Relay UE to which the Remote UE is connected. These assumptions ensure a stable environment for our model.

Lastly, we neglect processing times. These delays are probably not as significant as control channel loss probability and other phenomena but do exist. We assume that as soon as a UE has an intent to transmit, it attempts to do so.

These assumptions result in a conservative model that can handle worst-case scenarios, especially when the discovery pool resources are flooded by a large number of devices.

\subsection{Analytical Derivation}

\subsubsection{Markov Chain}

We use a Markov chain to model the system described in the previous section. We use an approach that is similar to the one in [6], but we consider the case when Remote UEs can transmit in different discovery periods. This creates different transmission groups that the model must consider, which results in the addition of an extra state to the Markov Chain. Although it might appear that the addition of a single state would not significantly affect the model, it significantly increases the complexity of the problem. 


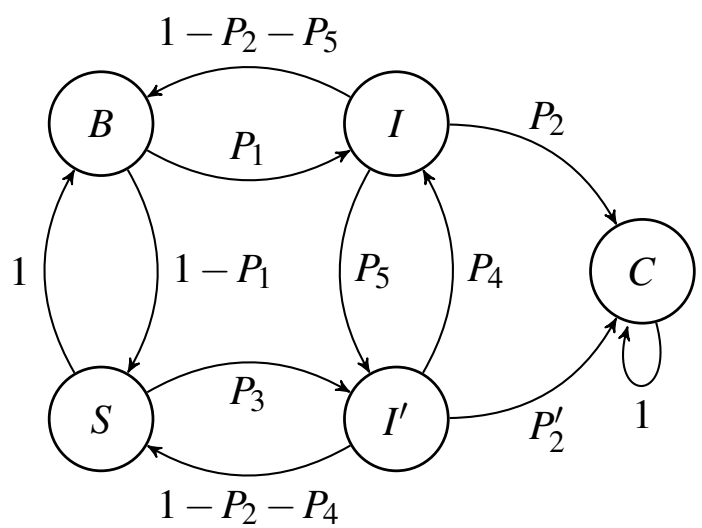

Fig. 2. Markov chain modeling the system in study.

Our Markov chain analysis requires that the sojourn times of all the states in our system follow a geometric probability distribution. While not all of the sojourn times do, they can be very closely approximated as geometrically distributed, so that the added complexity introduced by using a semi-Markov chain model that would capture the actual sojourn times is not worth the small gain in accuracy. Therefore, we have elected to use standard Markov chains in this analysis.

All Remote UEs have a sleeping period of 1, i.e., they alternate between active and sleep periods regardless of the outcome of their attempted transmission. Each Remote UE decides randomly and independently whether it starts transmitting in the first or second discovery period. We define $N_{x 1}=\left|G_{1}\right|$ to be the size of the group of UEs that transmits during the first period and $N_{x 2}=\left|G_{2}\right|$ to be the size of the group of UEs that sleep during the first period. Note that because $G_{1} \cap G_{2}=\varnothing, N_{x 1}+N_{x 2}=|G|$.

The states of our system are as follows:

- $B$ is the Beginning state. No solicitations are pending and the UEs in $G_{1}$ transmit.

- $S$ is the Sleeping state. UEs in $G_{1}$ are sleeping, and UEs in $G_{2}$ transmit. No successful solicitations have been issued.

- $I$ is the Intermediary state where solicitations have been issued to the set of Relay UEs, and UEs in $G_{2}$ transmit.

- $I^{\prime}$ is the Intermediary state where the UEs in $G_{1}$ are the next transmitting group.

- $C$ is the Completed state. It is reached when Remote UE $X$ successfully discovers Relay UE $Y$. It is the sole absorbing state of the system.

Because a Markov chain is memoryless, we cannot keep track of which Relay UEs have to attempt to respond to messages from the Remote UEs. Consequently, we assume that all Relay UEs attempt to respond to solicitation messages. The only way for a Relay UE not to receive a solicitation message while another Relay UE does is if it transmits in the 
subframe occupied by the solicitation message, assuming no other successful solicitations have been made outside of its sole subframe.

Recall that a Remote UE makes a successful solicitation when it transmits in an otherwise unoccupied resource block. A successful response entails not only the broadcast of a response message but also the half-duplex availability of the final receiver (UE $X$ in this context). Relay UEs do not queue received solicitations: their memory spans only to the previous period. The Markov chain's state transition probabilities are as follows:

- $P_{1}$ is the probability that at least one Remote UE in $G_{1}$ makes a successful solicitation to Relay UE $Y$.

- $P_{2}$ is the probability that Relay UE $Y$ responds successfully to Remote UE $X$, and its response message avoids collisions with solicitations from the Remote UEs in $G_{2}$.

- $P_{2}^{\prime}$ is the probability that Relay UE $Y$ responds successfully to Remote UE $X$, and its response message avoids collisions with solicitations from the Remote UEs in $G_{1}$.

- $P_{3}$ is the probability that at least one Remote UE in $G_{2}$ makes a successful solicitation to Relay UE $Y$.

- $P_{4}$ is the probability that no Relay UEs in $H$ send a response to Remote UE $X$, and Relay UE $Y$ simultaneously receives a new solicitation from at least one Remote UE in $G_{2}$.

- $P_{5}$ is the probability that no Relay UEs in $H$ send a response to Remote UE $X$ and Relay UE $Y$ simultaneously receives a new solicitation from at least one Remote UE in $G_{1}$.

The state transition matrix of our Markov chain is

$$
T=\left[\begin{array}{ccccc}
0 & 1-P_{1} & P_{1} & 0 & 0 \\
1-P_{3} & 0 & 0 & P_{3} & 0 \\
1-P_{2}-P_{5} & 0 & 0 & P_{5} & P_{2} \\
0 & 1-P_{2}^{\prime}-P_{4} & P_{4} & 0 & P_{2}^{\prime} \\
0 & 0 & 0 & 0 & 1
\end{array}\right] .
$$

Since $T$ is the state transition matrix of an absorbing Markov chain, we can partition it as follows, where $Q$ is a $4 \times 4$ matrix, $R$ is a length- 4 column vector, and 0 is a length- 4 all-zero row vector:

$$
T=\left[\begin{array}{cc}
Q & R \\
0 & 1
\end{array}\right]
$$

Using Eq. (2), we obtain the inverse of $T$ 's fundamental matrix, $N^{-1}=(1-Q)$ :

$$
N^{-1}=\left[\begin{array}{cccc}
1 & P_{1}-1 & -P_{1} & 0 \\
P_{3}-1 & 1 & 0 & -P_{3} \\
P_{5}+P_{2}-1 & 0 & 1 & -P_{5} \\
0 & P_{4}+P_{2}^{\prime}-1 & -P_{4} & 1
\end{array}\right] .
$$


We require only the first row of $N$ to derive our metric of interest. The mean time (in discovery periods) to go from state $B$ to $C$ is:

$$
\begin{aligned}
t(B \rightarrow C) & =\sum_{j=1}^{5} N_{1, j} \\
& =\frac{\left(1-P_{4} P_{5}\right)\left(2-P_{1}\right)+\left(1-P_{1}\right) P_{3}\left(1+P_{4}\right)+P_{y}\left(P_{3}-P_{1} P_{5}\right)+P_{1}\left(1+P_{5}+P_{3} P_{y}\right)}{\operatorname{det}(1-Q)}
\end{aligned}
$$

where

$$
\begin{aligned}
\operatorname{det}(1-Q)= & \left(1-P_{4} P_{5}\right)\left(1+\left(1-P_{1}\right)\left(P_{3}-1\right)\right) \\
& +P_{3}\left(P_{4} P_{x}-P_{5} P_{y}+P_{1} P_{x} P_{y}\right)+P_{1}\left(P_{x}-P_{5} P_{y}\left(P_{3}-1\right)\right)
\end{aligned}
$$

and where $P_{x}=P_{5}+P_{2}-1$ and $P_{y}=P_{4}+P_{2}^{\prime}-1$. We now derive the $P_{i}$ terms.

\subsubsection{State Transition Probabilities}

To get closed-form expressions for the state transition probabilities, we need the probability that a transmitted message reaches a receiver without colliding with other transmissions (both solicitations and responses). We use a combinatoric model, the classic "distinguishable balls in distinguishable bins," where the bins are discovery pool resource blocks and the balls are discovery message transmissions. We distinguish between the two types of discovery messages my assigning different colors to the balls in this model; we have $k$ balls, $j$ of which are black (the other $k-j$ being white, $k \leq j$ ) and $n$ bins.

While we are interested in the probability of successful transmissions, it is easier to obtain the probability of the complementary event (no successful transmissions). Since a successful transmission occurs if a discovery message does not collide with any other messages, we can abstractly represent collisions by events where multiple balls occupy a bin, and the balls sharing the bin thus represent colliding messages. To get the probability of no successful transmissions, we define $A_{j, k, n}$ to be the event: None of the j black balls out of $k$ balls is left alone in any of $n$ bins. Note that this event can occur if there is at least one bin containing multiple white balls.

Theorem 1. $P\left(A_{j, k, n}\right)$, the probability that event $A_{j, k, n}$ occurs, is

$$
P\left(A_{j, k, n}\right)=\frac{1}{n^{k}} \sum_{i=0}^{\min (j, n)}(-1)^{i}\left(\begin{array}{c}
n \\
i
\end{array}\right) \frac{j !}{(j-i) !}(n-i)^{k-i} .
$$

Proof. We number our bins 1 to $n$. Let $B_{r}$ be the event: Bin $r$ contains exactly one black ball. This event occurs if we place one black ball in bin $r$ and randomly distribute all other balls (black and white) in the remaining bins. There are $j$ potential balls we could have placed in bin $r$. For the other $k-1$ balls, there are $n-1$ bins left to choose from so there are $j(j-1)^{k-1}$ ways of placing the remaining balls. 
The same approach for the event $B_{r} \cap B_{s}$ with $r \neq s$ leads to $j(j-1)(n-2)^{k-2}$ possible arrangements of balls. For event $B_{r} \cap B_{s} \cap B_{t}$, there are $j(j-1)(j-2)(n-3)^{k-3}$ ways to place the remaining balls. Using the general form, we apply inclusion/exclusion to get

$$
\sum_{i=0}^{j}(-1)^{i}\left(\begin{array}{l}
n \\
i
\end{array}\right) \frac{j !}{(j-i) !}(n-i)^{k-i}
$$

combinations. Dividing Eq. (7) by the total number of arrangements, $n^{k}$, gives Eq. (6).

We start by deriving $P_{1}$ and $P_{3}$. Because Remote UEs broadcast all their solicitation messages to all Relay UEs, and any solicitation message can trigger a response message that Remote UE $X$ can receive, the forms of the expressions for $P_{1}$ and $P_{3}$ will be the same, but with $N_{x 1}$ in the expression for $P_{1}$ replaced by $N_{x 2}$ in the expression for $P_{3}$. We thus have a general formula for both $P_{1}$ and $P_{3}$, which is

$$
P_{\text {solicitation }}=P\left(G_{1} \rightarrow Y\right)=\sum_{k=0}^{N}(1-P(\text { All of } k \text { requests collide })) P(k \text { requests are sent })
$$

where $P_{\text {solicitation }}=P_{1}$ when $N=N_{x 1}$ and $P_{\text {solicitation }}=P_{3}$ when $N=N_{x 2}$. When our system is in states $B$ or $S$, no Relay UEs are transmitting. This scenario is similar to Model A, and we can use the formulas from [6] to obtain

$$
P_{\text {solicitation }}=\sum_{k=1}^{N}\left(\begin{array}{c}
N_{x} \\
k
\end{array}\right) \theta^{k}(1-\theta)^{N_{x}-k}\left[\sum_{i=1}^{\min \left(k, N_{x}\right)}(-1)^{i+1}\left(\begin{array}{c}
N_{x} \\
i
\end{array}\right)\left(\begin{array}{c}
k \\
i
\end{array}\right) i !\left(\frac{1}{N_{x}}\right)^{i}\left(1-\frac{i}{N_{x}}\right)^{k-i}\right]
$$

The response probabilities, $P_{2}$ and $P_{2}^{\prime}$, are also similar, but the response depends on Remote UE $X$ 's status. If Remote UE $X$ is in the sleeping state, it will be able to receive any non-collided response message, whereas if Remote UE $X$ is attempting to issue more queries, the half-duplex effect might prevent it from successfully receiving a response. Consequently:

$$
P_{2}=P(Y \sim X)=\theta\left(1-\frac{\theta}{N_{r}}\right)^{N_{y}+N_{x 2}-1}
$$

and

$$
P_{2}^{\prime}=\theta\left(1-\frac{\theta}{N_{t}}\right)\left(1-\frac{\theta}{N_{r}}\right)^{N_{y}+N_{x 1}-1}
$$

Next, $P_{4}=P\left((\overline{Y \sim X}) \cap\left(G_{1} \rightarrow Y\right)\right)$ and $P_{5}=P\left((\overline{Y \sim X}) \cap\left(G_{2} \rightarrow Y\right)\right)$ but the involved events are not independent. We solve this problem using conditional independence and 
obtain

$$
\begin{aligned}
P_{4}= & \sum_{j=1}^{N_{x 1}} \sum_{l=0}^{N_{y}-1}\left(\begin{array}{c}
N_{x 1} \\
j
\end{array}\right)\left(\begin{array}{c}
N_{y}-1 \\
l
\end{array}\right) \theta^{j+l}(1-\theta)^{N_{x 1}+N_{y}-1-j-l} \\
\times\left[(1-\theta)\left(1-P\left(A_{j, j+l, N_{r}}\right)\right)+\theta\right. & \sum_{k=0}^{j} \sum_{m=0}^{l}\left(\begin{array}{l}
j \\
k
\end{array}\right)\left(\begin{array}{c}
l \\
m
\end{array}\right)\left(\frac{1}{N_{t}}\right)^{k+m}\left(1-\frac{1}{N_{t}}\right)^{l+j-k-m} \\
& \left.\times P\left(A_{1, k+m+1, N_{f}}\right)\left(1-P\left(A_{j-k, j+l-k-m, N r-N f}\right)\right)\right] .
\end{aligned}
$$

We derive the expression for $P_{5}$ by conditioning on the Remote UE $X$ 's status to account for the half-duplex effect.

$$
\begin{aligned}
& P_{5}=\sum_{j=0}^{N_{x 2}} \sum_{l=0}^{N_{y}-1}\left[\left(\begin{array}{c}
N_{x 2} \\
j
\end{array}\right)\left(\begin{array}{c}
N_{y}-1 \\
l
\end{array}\right) \theta^{j+l}(1-\theta)^{N_{x 2}+N_{y}-j-l}\left(1-P\left(A_{j, j+l, N_{r}}\right)\right)\right] \\
& +\sum_{j=0}^{N_{x 2}-1} \sum_{l=0}^{N_{y}-1}\left\{\left(\begin{array}{c}
N_{x 2}-1 \\
j
\end{array}\right)\left(\begin{array}{c}
N_{y}-1 \\
l
\end{array}\right) \theta^{j+l+1}(1-\theta)^{N_{x 2}+N_{y}-j-l-2}\right. \\
& \times\left(( 1 - \theta ) \sum _ { k = 0 } ^ { j } \sum _ { m = 0 } ^ { l } \left[\left(\begin{array}{l}
j \\
k
\end{array}\right)\left(\begin{array}{l}
l \\
m
\end{array}\right)\left(\frac{1}{N_{t}}\right)^{k+m}\left(1-\frac{1}{N_{t}}\right)^{j+l-k-m}\right.\right. \\
& \times\left[\left(1-P\left(A_{j-k, j-k+l-m, N_{r}-N_{f}}\right)\right)\left(P\left(A_{1, k+m+1, N_{f}}\right)+\theta / N_{t}\right)\right. \\
& \left.\left.\left.\left.+\left(1-P\left(A_{j-k+1, j-k+l-m+1, N_{r}-N_{f}}\right)\right) \theta\left(1-\frac{1}{N_{t}}\right) P\left(A_{1, k+m+1, N_{f}}\right)\right]\right]\right)\right\} .
\end{aligned}
$$

We can now compute $t(X \circ Y)$, the mean number of discovery periods required for a Remote UE to go from the beginning state $B$ to the completed state $C$ given in Eq. (4) and (5). We use the expressions for $P_{1}, P_{3}, P_{2}, P_{2}^{\prime}, P_{4}$, and $P_{5}$ from Eq. (9), (10), (11), and (12), where Eq. (11) and (12) rely on the expression for $P\left(A_{j, k, n}\right)$ in Eq. (6). For each set of parameter values that we consider, we evaluate the state transition probabilities for all possible values of $\left|G_{1}\right|$ and $\left|G_{2}\right|$ that satisfy $\left|G_{1}\right|+\left|G_{2}\right|=|G|$ and take the average over the entire set. We can do this because UEs choose their respective transmission patterns independently, which makes all combinations equally likely. If one group happens to be empty, then Model B reduces to the simplified model described in [6]. 


\section{Numerical Results}

\subsection{System-Level Simulations}

We validated the analytical model of the previous section using system-level simulations. We used the ns-3 ProSe model presented in [7] and extended with the UE-to-Network Relay capability as described in [4]. We configured $N_{y}$ Relay UEs and $N_{x}$ Remote UEs using direct discovery Model B with the same discovery pool parameters $\left(N_{f}, N_{t}\right.$, txProbability) and a discovery period of $320 \mathrm{~ms}$. The Remote UEs send discovery solicitations every other period, and the first solicitation for each Remote UE is generated randomly in a range of two discovery periods to ensure interleaving.

In order to have a fair validation and match the assumptions made for the analytical model, we considered ideal channel conditions and that discovery messages sent by multiple UEs in the same discovery pool resource are dropped. In the simulations, a Remote UE discovers a Relay UE when the former successfully receives and decodes a response message from the latter. In each trial, we calculated the metric of interest, $t(X \circ Y)$, for a randomly chosen Remote UE, $X$, and a randomly chosen Relay UE, $Y$. We performed 1000 independent trials for each configuration, and all results are presented showing the mean values together with $95 \%$ confidence intervals.

\subsection{Result Discussion}

We obtained the results shown in Fig. 3 for a discovery resource pool spanning $N_{f}=2$ PRBs and $N_{t}=5$ subframes, and we tried all values defined in the standard for the $t x$ Probability parameter, i.e., $0.25,0.50,0.75$, and 1.0. The results show close agreement between the system-level simulations and the analytical model. Although we show results only for three values of $N_{x}$, the trends for higher and lower values of $N_{x}$ are coherent and match systemlevel simulations.

The trends we observed conform to our understanding of the problem: more UEs contending for resources results in longer average discovery times. The growth of $t(X \circ Y)$ also depends on the flooding of the discovery pool's resources; i.e., for low values of txProbability the increase is relatively small, leading to a quasi-linear evolution, whereas high values of txProbability and $N_{x}$ cause exponential growth of the mean discovery time. A natural exception to this observation occurs when either $G_{1}$ or $G_{2}$ are empty, in which case the scenario reduces to the one in [6], where there is no contention for resources between Relay UEs and Remote UEs.

We generated these results for a fixed resource pool ( $N_{f}=2$ PRBs; $N_{t}=5$ subframes). Smaller resource pools would make smaller values of the input parameters produce exponentially increasing values of $t(X \circ Y)$ versus $N_{y}$, whereas larger pools would lead quasilinear increases in $t(X \circ Y)$ over larger sets of values of $N_{y}$. Increasing $N_{t}$ (i.e., adding subframes to the pool) would lead to a decrease of message losses due to the half-duplex

effect, thus decreasing mean discovery times proportionally to the population of UEs using 


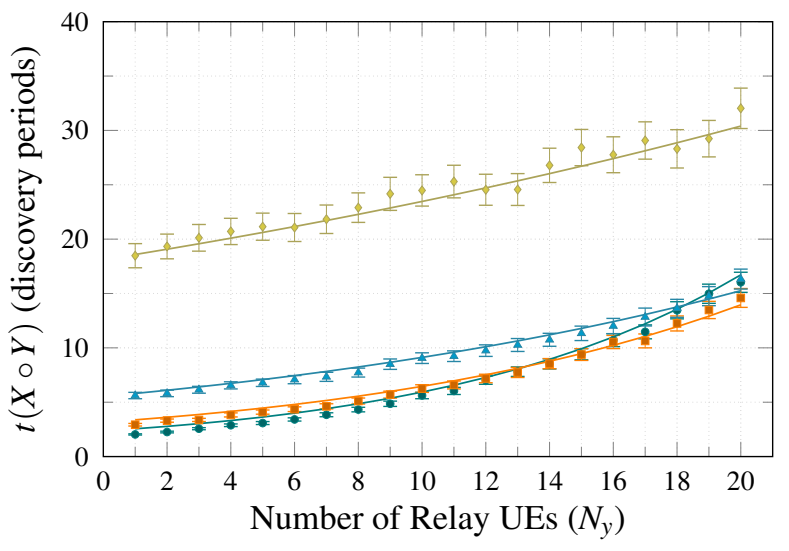

- Simulation, txProbability $=1.00$

- Simulation, $t x$ Probability $=0.75$

- Simulation, $t x$ Probability $=0.50$

- Simulation, txProbability $=0.25$

- Analytical, txProbability $=1.00$

- Analytical, txProbability $=0.75$

- Analytical, txProbability $=0.50$

- Analytical, txProbability $=0.25$

(a) $N_{x}=2$ Remote UEs

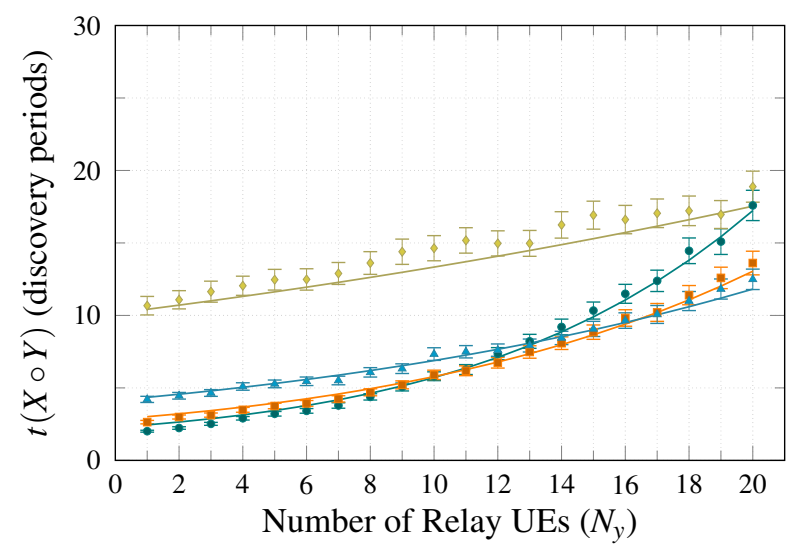

- Simulation, txProbability $=1.00$

- Simulation, txProbability $=0.75$

- Simulation, $t x$ Probability $=0.50$

- Simulation, $t x$ Probability $=0.25$

- Analytical, txProbability $=1.00$

- Analytical, txProbability $=0.75$

- Analytical, txProbability $=0.50$

- Analytical, txProbability $=0.25$

(b) $N_{x}=5$ Remote UEs

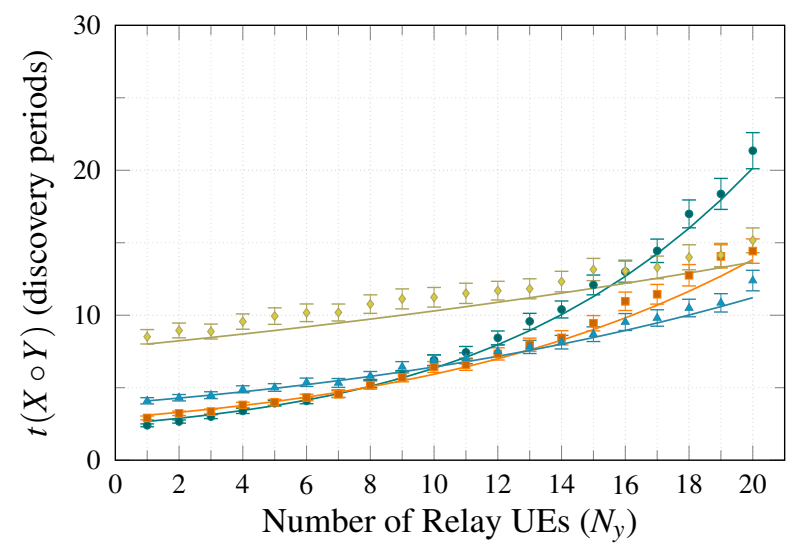

- Simulation, $t x$ Probability $=1.00$

- Simulation, txProbability $=0.75$

$\triangle$ Simulation, $t x$ Probability $=0.50$

- Simulation, txProbability $=0.25$

- Analytical, txProbability $=1.00$

- Analytical, txProbability $=0.75$

- Analytical, txProbability $=0.50$

- Analytical, txProbability $=0.25$

(c) $N_{x}=10$ Remote UEs

Fig. 3. Average number of discovery periods needed by a Remote UE to discover a given Relay UE $(t(X \circ Y))$. Mean and $95 \%$ confidence intervals are shown for the system-level simulation results. 
the pool. Increasing $N_{f}$, (i.e., adding PRB pairs) would spread query messages in frequency but not in time, leading to a greater half-duplex effect and greater mean discovery times. However, as shown in [6], the increase of the discovery pool's size enhances the performance negligibly after reaching a cap but comes at the expense of used resources (time and frequency) that could be more effective if allocated elsewhere.

One of the most interesting features to observe is the crossing point between the $t(X \circ Y)$ curves. This point indicates the value of $N_{y}$ for which it is better to choose lower values of $t x$ Probability. In Fig. $3 b$ we observe that it is always better to pick txProbability $=1.0$ rather than $t x$ Probability $=0.25$ as long as we have no more than 20 Relay UEs transmitting. The break point between those values drops down to $N_{y}=16$ for $N_{x}=10$ in Fig. $3 \mathrm{c}$ and continues dropping down when we increase $N_{x}$. Lower values of $N_{x}$ and $N_{y}$ produce longer mean discovery times when we use lower values of txProbability because of less frequent transmissions. In those cases, we have less contention and unused pool resources, meaning that could be better to opt for more sustained transmissions.

Finally, we note that the analytical model results are mostly less than the simulation results' mean values, especially for $t x$ Probability $=0.25$. We attribute this to two factors. First, the model does not account for all the layers of the network that the simulations do, and this additional delay may be caused by higher layers. The second and more important factor is that we assume that all Relay UEs respond to a successful solicitation message; this neglects the fact that some Relay UEs might not receive the solicitation message due to the half-duplex effect. This also explains why the theoretical model's output is less than the simulation's output even for higher values of txProbability.

\section{Conclusions and Future Work}

In this paper, we presented an efficient approach to analyze Model B direct discovery in ProSe-enabled networks using UE-to-Network Relays. The results show the relative influence of each parameter, supported by system-level simulations. Because our theoretical model can provide results on the order of minutes for the sets of parameters, it is a practical tool for assessing large sets of configurations for various use cases. More in-depth conclusions can be made by setting particular sets of conditions to reflect desired use cases.

Future work on the subject could extend this approach to other metrics of the discovery process, such as the time for a Remote UE to find any Relay UE, or all Relay UEs in its range. Further improvements could include adding packet recovery from collisions, analysis of non-optimal conditions, incorporating the effect of higher layers, or even the topology of the network of UEs. 


\section{References}

[1] Griffith D, Lyons F (2016) Optimizing the UE Transmission Probability for D2D Direct Discovery. 2016 IEEE Global Communications Conference (GLOBECOM), , pp 1-6. https://doi.org/10.1109/GLOCOM.2016.7842110

[2] 3GPP (2018) Technical Specification Group Services and System Aspects; Proximitybased services (ProSe); Stage 2 (Release 15) (3GPP), TS 23.303 v15.1.0.

[3] 3GPP (2018) Technical Specification Group Core Network and Terminals; Proximityservices (ProSe) User Equipment (UE) to ProSe function protocol aspects; Stage 3 (Release 15) (3GPP), TS 24.334 v15.2.0.

[4] Gamboa S, Thanigaivel R, Rouil R (2019) System Level Evaluation of UE-to-Network Relays in D2D-Enabled LTE Networks. 2019 IEEE 24th International Workshop on Computer Aided Modeling and Design of Communication Links and Networks (CAMAD), , pp 1-7. https://doi.org/10.1109/CAMAD.2019.8858441

[5] 3GPP (2018) Technical Specification Group Radio Access Network; Evolved Universal Terrestrial Radio Access (E-UTRA); Radio Resource Control (RRC); Protocol specification (Release 15) (3GPP,), TS 36.331 v15.3.0.

[6] Gamboa S, Moreaux A, Griffith D, Rouil R (2020) UE-to-Network Relay Discovery in ProSe-enabled LTE Networks. 2020 International Conference on Computing, Networking and Communications (ICNC), , pp 871-877. https://doi.org/10.1109/ ICNC47757.2020.9049657

[7] Rouil R, Cintrón FJ, Ben Mosbah A, Gamboa S (2017) Implementation and Validation of an LTE D2D Model for Ns-3. Proceedings of the Workshop on ns-3 WNS3 '17, , p 55-62. https://doi.org/10.1145/3067665.3067668 